\title{
One-dimensional spinon spin currents
}

\author{
Daichi Hirobe $^{1 \star}$, Masahiro Sato ${ }^{2,3 \dagger}$, Takayuki Kawamata ${ }^{4}$, Yuki Shiomi ${ }^{1,2}$, Ken-ichi Uchida ${ }^{1,5}$, \\ Ryo Iguchi ${ }^{1,2}$, Yoji Koike ${ }^{4}$, Sadamichi Maekawa ${ }^{2,3}$ and Eiji Saitoh ${ }^{1,2,3,6 \star}$
}

\begin{abstract}
Quantum spin fluctuation in a low-dimensional or frustrated magnet breaks magnetic ordering while keeping spin correlation'. Such fluctuation has been a central topic in magnetism because of its relevance to high- $T_{\mathrm{c}}$ superconductivity ${ }^{2}$ and topological states ${ }^{3}$. However, utilizing such spin states has been quite difficult. In a one-dimensional spin-1/2 chain, a particle-like excitation called a spinon is known to be responsible for spin fluctuation in a paramagnetic state. Spinons behave as a Tomonaga-Luttinger liquid at low energy, and the spin system is often called a quantum spin chain ${ }^{4}$. Here we show that a quantum spin chain generates and carries spin current, which is attributed to spinon spin current. This is demonstrated by observing an anisotropic negative spin Seebeck effect ${ }^{5-10}$ along the spin chains in $\mathrm{Sr}_{2} \mathrm{CuO}_{3}$. The results show that spin current can flow even in an atomic channel owing to long-range spin fluctuation.
\end{abstract}

The flow of electron spins is defined as spin current. In condensed matter science, the transport properties of spin current have attracted considerable interest since the discovery of various spincurrent phenomena ${ }^{11,12}$. However, in spintronics ${ }^{13}$, finding materials that can carry spin angular momentum efficiently is of critical importance, especially for integrated microscopic devices.

So far, two types of spin current, conduction-electron spin current and spin-wave spin current, have experimentally been explored. The former is mediated by the motion of electrons in metals and semiconductors. Thus, its velocity and propagation length are limited by electron spin diffusion. In the latter type ${ }^{14,15}$, spin waves, wave-like propagation of spin motions in magnets, carry spin angular momentum. Its excitation gap is equal to a spin-wave gap, proportional to the magnetic anisotropy. In addition, spin-wave spin current can exist even in insulators in which spin relaxation via conduction electrons is absent. This is an advantage that may realize fast and long-range spin-current transmission, opening a new field of insulator-based spintronics. However, spin-wave spin current in classical magnets may not be suitable for microscopic devices, since handling spin waves becomes difficult when devices are miniaturized toward atomic scale. In ferromagnets, spontaneous magnetization brings about significant stray fields, causing crosstalk. However, in antiferromagnetic systems, spin ordering patterns should be broken or interfered when a device is in atomic scale; in both these cases, spin waves become vulnerable. Therefore, to realize spin-current transport in microscopic devices, spin ordering is expected to vanish while maintaining a strong interaction between spins.

Here, we would like to introduce a new type of spin current: spinon spin current, which may provide a channel for spin transmission based on quantum fluctuation without spin ordering. Spinons generally refer to magnetic elementary excitations in quantum spin liquids ${ }^{1}$. When the system size of a magnet is reduced to atomic scale, quantum spin fluctuation manifests itself and dominates spin properties. The most typical example is found in one-dimensional spin-1/2 chains realized in some oxides, such as an insulator $\mathrm{Sr}_{2} \mathrm{CuO}_{3}$ (refs 16-18). In $\mathrm{Sr}_{2} \mathrm{CuO}_{3}$, each $\mathrm{Cu}^{2+}$ ion carries spin-1/2 and they are connected to each other linearly along the $b$ axis, as shown in Fig. 1a. Because of the one-dimensionality, the fluctuation of the spin- $1 / 2$ is so strong that it prevents magnetic ordering. As a result, antiferromagnetic interaction embedded in the chain creates a paramagnetic state accompanied by strong spinsinglet correlation. Spin excitation from the correlated ground state has been predicted to be particle-like and to exhibit zero excitation gap, and this excitation is known as a spinon. This gapless feature is robust against external magnetic fields and magnetic anisotropy. Furthermore, theories have predicted that the correlation of spinons is of a markedly long length scale; even infinite correlation length is predicted in the context of the Tomonaga-Luttinger (TL) liquid theories ${ }^{4}$. These indicate that in such a system spin current may propagate through a long distance via spinons.

To drive the spin current, one of the most versatile methods is to use a longitudinal spin Seebeck effect ${ }^{7,8,10}$ (LSSE). LSSE refers to the generation of spin current as a result of a temperature gradient applied across the junction between a magnet, typically a magnetic insulator, and a metal film, typically Pt. The temperature gradient injects spin current into the metal from the magnet. The injected spin current is converted into electric voltage via the inverse spin Hall effect ${ }^{19-21}$ (ISHE) in the metal. The voltage is generated perpendicular to the spin polarization and the propagation directions of the spin current. By measuring the voltage generated, the method enables sensitive detection of spin current. The amplitude of the injected spin current is proportional to the non-equilibrium accumulation of spin angular momentum at the interface in the magnet. In this study, we utilized the LSSE to extract spin current from a spinon system.

Spinon-induced LSSE is characterized by a distinguished feature: theory predicts that the sign of angular momentum due to a spinon LSSE is opposite to that of the conventional spin-wave-induced LSSE at low temperatures under magnetic fields. The opposite spin angular momentum is mainly due to the singlet correlation increasing with decreasing temperature in the present spinon system in contrast to ferromagnetic correlation growing in classical magnets. Detailed theoretical calculations using a microscopic model reproduce this intuition, which is described below. By exploiting these properties of LSSE, we observed spin-current

\footnotetext{
${ }^{1}$ Institute for Materials Research, Tohoku University, Sendai 980-8577, Japan. ${ }^{2}$ Spin Quantum Rectification Project, ERATO, Japan Science and Technology Agency, Sendai 980-8577, Japan. ${ }^{3}$ The Advanced Science Research Center, Japan Atomic Energy Agency, Tokai 319-1195, Japan. ${ }^{4}$ Department of Applied Physics, Tohoku University, Sendai 980-8579, Japan. ${ }^{5}$ PRESTO, Japan Science and Technology Agency, Saitama 332-0012, Japan. ${ }^{6}$ WPI Advanced Institute for Materials Research, Tohoku University, Sendai 980-8577, Japan. †Present address: Department of Physics, Ibaraki University, Mito, Ibaraki310-8512, Japan. *e-mail: daichi.kinken@imr.tohoku.ac.jp; eizi@imr.tohoku.ac.jp
} 
a

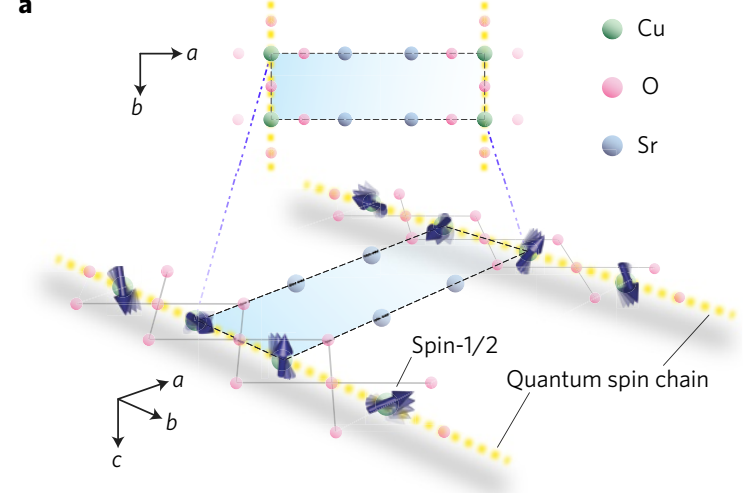

b

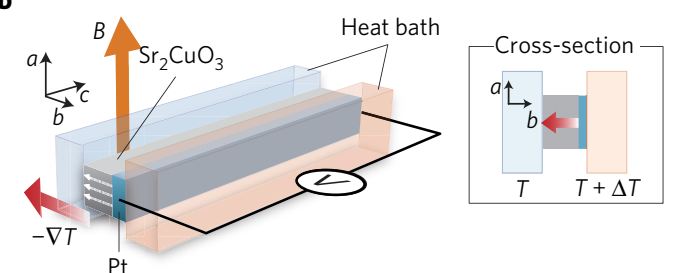

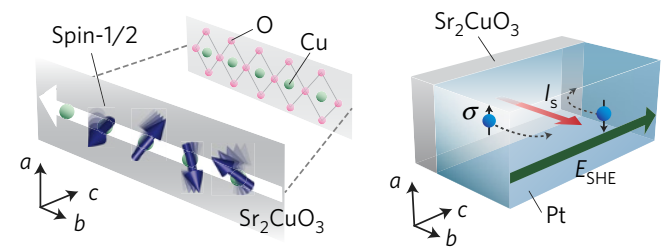

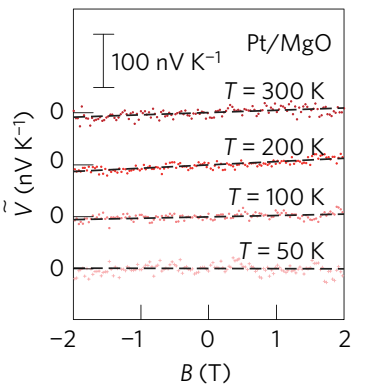

d

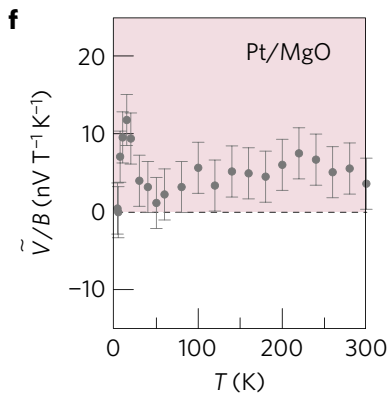

Figure 1 | Quantum spin chain, experimental set-up, and thermoelectric response of Pt film. a, Quantum spin chains in $\mathrm{Sr}_{2} \mathrm{CuO}_{3}$. Quantum spin chains along the $b$ axis consist of $\mathrm{Cu}^{2+}$ ions sharing $\mathrm{O}^{2-}$ ions. $\mathbf{b}$, Schematic illustration of the experimental set-up. The sample consists of a single-crystalline $\mathrm{Sr}_{2} \mathrm{CuO}_{3}$ and a Pt film. A temperature gradient, $\nabla T$, is generated along the spin chains ( $b$ axis) by applying a temperature difference, $\Delta T$. $B$ denotes an external magnetic field and $T$ the system temperature. c, Configuration of quantum spin chains in the experimental set-up. A Cu-O chain is set along $\nabla T$. d, Schematic illustration of the inverse spin Hall effect. An electric field, $E_{\mathrm{SHE}}$, arises in the direction of $I_{\mathrm{s}} \times \sigma$ in Pt. Here $I_{\mathrm{s}}$ and $\sigma$ are the spatial direction and the spin-polarization direction of spin current, respectively. $\mathbf{e}, B$ dependence of $\widetilde{V}=V / \Delta T$, the voltage $V$ divided by the temperature difference $\Delta T$, in a Pt film put on a single-crystalline $\mathrm{MgO}$. The dashed lines are fits to the data points. $\mathbf{f}, T$ dependence of $\widetilde{V} / B$ measured in Pt/MgO. The error bars represent the $68 \%$ confidence level ( \pm s.d.).

generation and transmission in $\mathrm{Sr}_{2} \mathrm{CuO}_{3}$, a typical paramagnetic insulator in which the spinon picture has well been established ${ }^{1}$.

Figure $1 \mathrm{~b}$ is a schematic illustration of the experimental setup used in this study. The sample consists of a single crystal of $\mathrm{Sr}_{2} \mathrm{CuO}_{3}$ and a $\mathrm{Pt}$ thin film. The Pt film is used as a spincurrent detector based on ISHE, in which spin current is converted into an electromotive force, $E_{\mathrm{SHE}}$ (Fig. 1d). The spin chains in $\mathrm{Sr}_{2} \mathrm{CuO}_{3}$ are set normal to the $\mathrm{Pt}$ film plane (Fig. 1c). A temperature gradient, $\nabla T$, was generated along the spin chains by applying the temperature difference $\Delta T$ between the top of the Pt film and the bottom of $\mathrm{Sr}_{2} \mathrm{CuO}_{3}$ (see also Fig. 1b). The voltage difference $V$ is measured between the ends of the Pt film while applying an in-plane field, $B$.

First, we measured the $\Delta T$-induced voltage in a $\mathrm{Pt}$ film without $\mathrm{Sr}_{2} \mathrm{CuO}_{3}$. In this simple film, the voltage is produced via the normal Nernst effect of Pt alone ${ }^{22}$. In Fig. 1e, we show the magnetic field $B$ dependence of the voltage at several temperatures. The voltage $\widetilde{V}=V / \Delta T$ (the voltage $V$ divided by the temperature difference $\Delta T$ ) was found to be proportional to $B$. In Fig. 1f, we show the temperature $T$ dependence of the slope $\widetilde{V} / B$ (that is, the Nernst coefficient of Pt). The sign of $\widetilde{V} / B$ is positive for the entire range of $T$, showing that the sign of the normal Nernst effect of Pt is positive in the whole temperature range in the present set-up.

The temperature dependence of $\widetilde{V} / B$ for Pt changes dramatically when $\mathrm{Sr}_{2} \mathrm{CuO}_{3}$ is attached to Pt. Figure 2a shows the $T$ dependence of $\widetilde{V} / B$ for $\mathrm{Pt} / \mathrm{Sr}_{2} \mathrm{CuO}_{3}$. The sign of $\widetilde{V} / B$ is positive around room temperature, the same sign as the normal Nernst effect in the simple Pt film. With decreasing $T$, surprisingly, the sign of $\widetilde{V} / B$ reverses around $180 \mathrm{~K}$ and is negative below this temperature (see also Fig. 2c,d). In addition, another sign reversal takes place with decreasing $T$ further and $\widetilde{V} / B$ changes to positive below about $5 \mathrm{~K}$, which is comparable to the Néel temperature of $\mathrm{Sr}_{2} \mathrm{CuO}_{3}$ (ref. 16). These sign reversals show that the negative $\widetilde{V} / B$ component appears by attaching the insulator $\mathrm{Sr}_{2} \mathrm{CuO}_{3}$ and that it dominates at low temperatures, but disappears following the Néel ordering. The negative sign of $\widetilde{V} / B$ cannot be explained by the normal Nernst effect of $\mathrm{Pt}$, but it is the very feature of the aforementioned spinon LSSE. On the other hand, the sign of the LSSE voltage for $\mathrm{Pt} /$ conventional ferromagnets, ferrimagnets or antiferromagnets is the same as that of the normal Nernst effect of $\mathrm{Pt}$ in the present set-up ${ }^{7,8,23}$. The sign reversal around $180 \mathrm{~K}$ is attributed to a competition between such a negative $\widetilde{V} / B$ component and the positive component due to the normal Nernst effect of Pt.

The sign reversal of $\widetilde{V} / B$ was found to be related to spin-current injection from $\mathrm{Sr}_{2} \mathrm{CuO}_{3}$ as follows. In Fig. 3a, $\widetilde{V} / B$ measured for $\mathrm{W} / \mathrm{Sr}_{2} \mathrm{CuO}_{3}$ is plotted as a function of $T$ (red data points), where $\mathrm{W}$ exhibits negative ISHE; the sign of ISHE of $\mathrm{W}$ is opposite to that of $\mathrm{Pt}$ (ref. 24). In $\mathrm{W} / \mathrm{Sr}_{2} \mathrm{CuO}_{3}, \widetilde{V} / B$ is always positive and does not exhibit any sign reversal (see also Fig. $3 c, d$ ), and remarkably, a $V / \Delta T$ peak with positive sign appears around $20 \mathrm{~K}$ (pink arrow in Fig. 3a): the opposite peak sign to that of $\mathrm{Pt} / \mathrm{Sr}_{2} \mathrm{CuO}_{3}$ (blue arrow in Fig. 3a). The sign change between $\mathrm{W}$ and $\mathrm{Pt}$ shows that the low-temperature $V / \Delta T$ signal is attributed mainly to ISHE due to spin current injected from $\mathrm{Sr}_{2} \mathrm{CuO}_{3}$. We note that the peak temperature $\sim 20 \mathrm{~K}$ is not the same as the temperature at which the spin thermal conductivity reaches its maximum $(\sim 50 \mathrm{~K}$ for a high-purity $\mathrm{Sr}_{2} \mathrm{CuO}_{3}$; ref. 25); similar results were reported for conventional spin waves in ferrimagnet $\mathrm{Y}_{3} \mathrm{Fe}_{5} \mathrm{O}_{12}$ (refs 10,26).

In Fig. $3 e, V / B$ was compared between the $\nabla T \| b$-axis and the $\nabla T \perp b$-axis configurations. The $b$ axis is the spin-chain direction of $\mathrm{Sr}_{2} \mathrm{CuO}_{3}$, and thus in the $\nabla T \| b$-axis $(\nabla T \perp b$-axis) configuration, the heat current flows parallel (normal) to the spin chains. Clearly, the negative $\widetilde{V} / B$ peak observed in $\mathrm{Pt} / \mathrm{Sr}_{2} \mathrm{CuO}_{3}$ is suppressed when $\nabla T \perp b$ axis: the amplitude of $\widetilde{V} / B$ at $20 \mathrm{~K}$ is one order of magnitude less than that in the $\nabla T \| b$-axis configuration (see also Fig. 3g,h). The suppression was confirmed also in $\mathrm{W} / \mathrm{Sr}_{2} \mathrm{CuO}_{3}$ (see the inset 
a

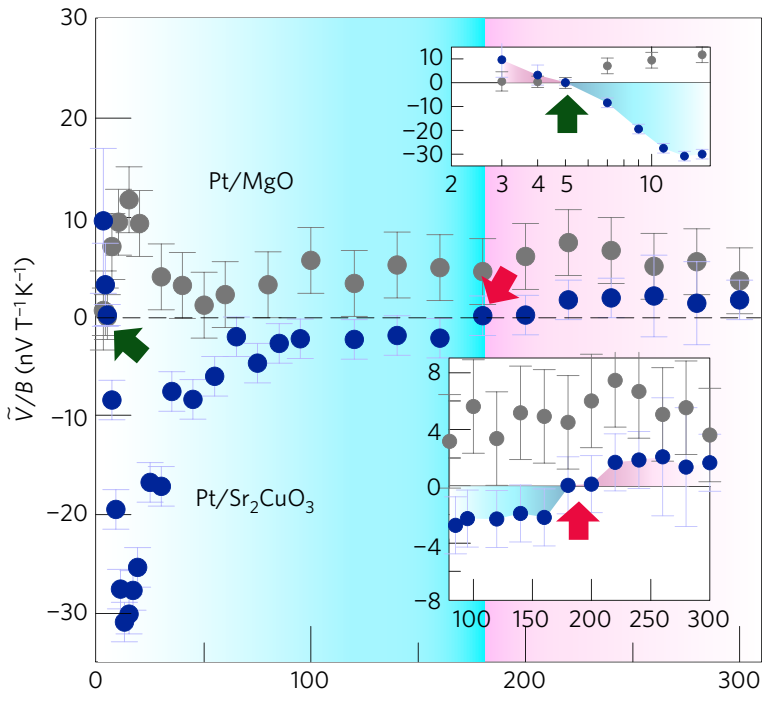

b

$T(\mathrm{~K})$

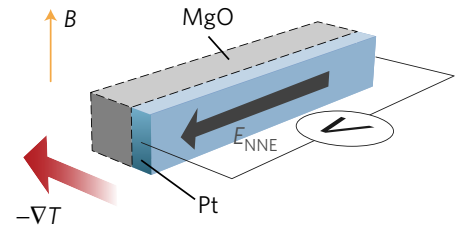

$\mathrm{Pt}_{2} \mathrm{Sr}_{2} \mathrm{CuO}_{3}$

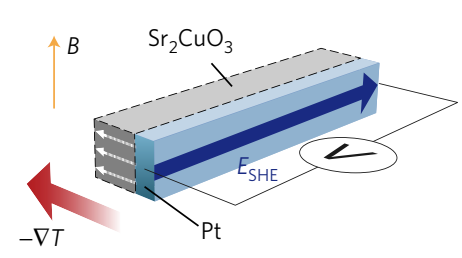

c

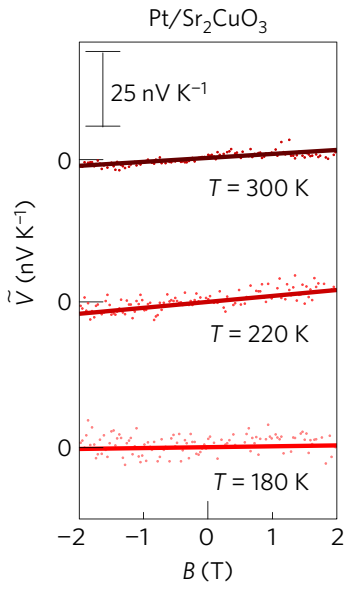

d

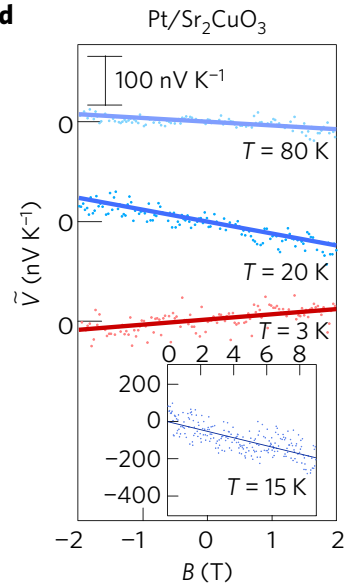

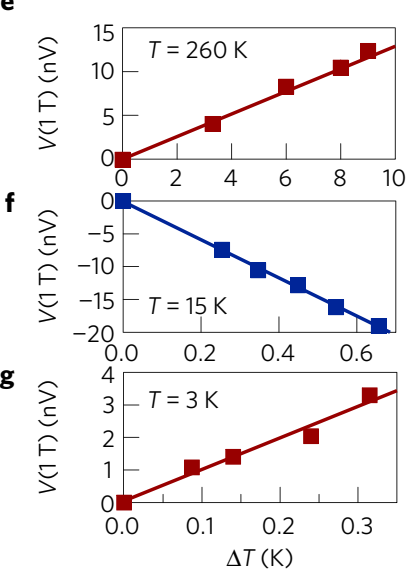

Figure 2 | Observation of negative spin Seebeck effect in $\mathrm{Pt}_{/} \mathrm{Sr}_{2} \mathrm{CuO}_{3} . \mathbf{a}, T$ dependence of $\widetilde{V} / \mathrm{B}$ measured in $\mathrm{Pt} / \mathrm{Sr}_{2} \mathrm{CuO}$. The data for Pt/MgO are also presented (see also Fig. 1f). The error bars represent the $68 \%$ confidence level ( \pm s.d.). The lower and upper insets show sign reversal at about $180 \mathrm{~K}$ and $5 \mathrm{~K}$, respectively. b, Experimental set-ups. In Pt/MgO (upper panel), the electric field $\mathrm{E}_{\mathrm{NNE}}$ arises via the normal $\mathrm{Nernst}$ effect in $\mathrm{Pt}$. In Pt/Sr2 $\mathrm{CuO} 3$ (lower panel), the electric field $E_{S H E}$ also arises via the inverse spin Hall effect in Pt. c,d, $B$ dependence of $\widetilde{V}$ measured in $\mathrm{Pt} / \mathrm{Sr}_{2} \mathrm{CuO} \mathrm{O}_{3}$ at various temperatures. The lines are fits to the data points. The inset to $\mathbf{d}$ shows the data taken up to $9 \mathrm{~T}$ at $15 \mathrm{~K}$. e-g, Dependence of $V$ on $\Delta T$ at $260 \mathrm{~K}(\mathbf{e}), 15 \mathrm{~K}(\mathbf{f})$ and $3 \mathrm{~K}(\mathbf{g})$ at $1 \mathrm{~T}$. In all of these cases, $V$ is proportional to $\Delta T$.

to Fig. 3e). The result shows that the spin-current injection from $\mathrm{Sr}_{2} \mathrm{CuO}_{3}$ takes place only when heat current is applied along the spin chain; the spin angular momentum flowing along the spin chains of $\mathrm{Sr}_{2} \mathrm{CuO}_{3}$ dominates the spin-current injection observed in the present study. The small negative signal of $\widetilde{V} / B$ for the $\nabla T \perp b$ axis (Fig. 3e) might be attributed to an inevitable slight misalignment in the $\nabla T$ direction from the $b$ axis $\left(\leq 6^{\circ}\right)$. We also note that the thermal conductivity and magnetic susceptibility of $\mathrm{Sr}_{2} \mathrm{CuO}_{3}$ are almost isotropic along the $a$ and $b$ axes ${ }^{18}$, and therefore, the voltage suppression cannot be attributed to a reduction in the magnitude of either $\nabla T$ or the field-induced magnetic moment. The negative and anisotropic LSSE is evidence that spin current is generated and conveyed by spinons through the spin chains of $\mathrm{Sr}_{2} \mathrm{CuO}_{3}$. Although seemingly similar results were reported for several three-dimensional materials, or $\mathrm{Gd}_{3} \mathrm{Fe}_{5} \mathrm{O}_{12}$ and $\mathrm{DyScO}_{3}$, the origins are completely different from that of the spinon LSSE. The negative sign of LSSE observed in the ferrimagnet $\mathrm{Gd}_{3} \mathrm{Fe}_{5} \mathrm{O}_{12}$ is attributed to competition in the spin injection between the different ordered magnetic ions ${ }^{27}$; in contrast, the magnetic ions of $\mathrm{Sr}_{2} \mathrm{CuO}_{3}$ are $\mathrm{Cu}^{2+}$ alone along with spin fluctuation down to $\sim 5 \mathrm{~K}$. Additionally, the anisotropic LSSE in a paramagnetic state of the antiferromagnet $\mathrm{DyScO}_{3}$ is due simply to the strong anisotropy of the magnetic susceptibility while an origin of the negative sign remains to be elucidated ${ }^{22}$.

Finally, we turn to a theoretical formulation of the spinon LSSE in the present system. On the basis of a microscopic theory for LSSE ${ }^{28}$ (see Supplementary Sections A and B), the spin current $I_{\mathrm{s}}$ injected from a magnet to a metal is given by

$$
I_{\mathrm{s}}=-\frac{A}{T^{2}} \int_{-\infty}^{\infty} \mathrm{d} \omega \operatorname{Im} \chi^{-+}(\omega) \operatorname{Im} X^{-+}(\omega) \frac{\delta T}{\sinh ^{2}(\omega /(2 T))}
$$

where $\omega$ is the angular frequency, $\delta T$ is the temperature difference between the magnet and the metal, $T$ is the mean value of the two temperatures, and $A$ is a material-dependent factor (see Supplementary Section B). $X^{-+}$and $\chi^{-+}$denote dynamical spin susceptibilities of the magnet and the metal, respectively. This formula can be applied to various magnetic phases (either magnetically ordered or disordered). We note that phenomenological transport theories for $\operatorname{LSSE}^{9}$ have also been developed and applied to ordered magnets, and in the formula (1), spinon coherent dynamics with a long mean free path is taken into account via the 


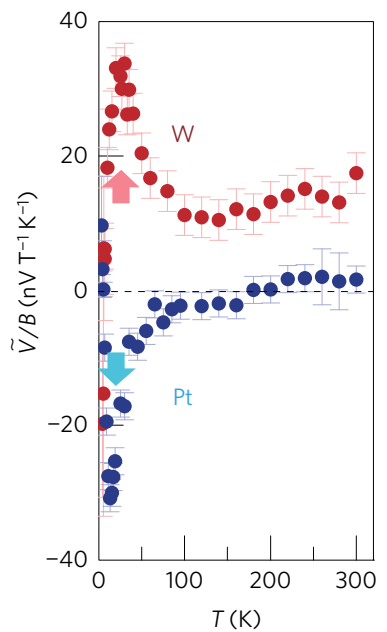

c

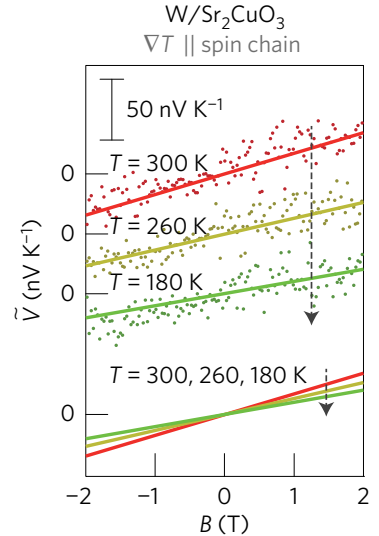

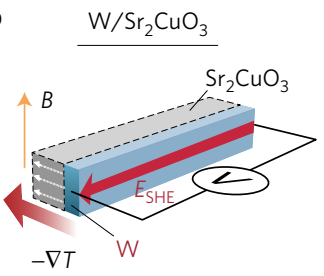

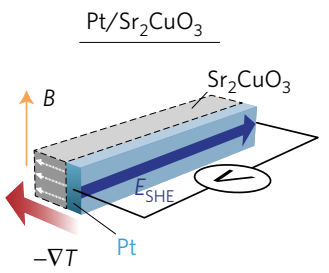

d

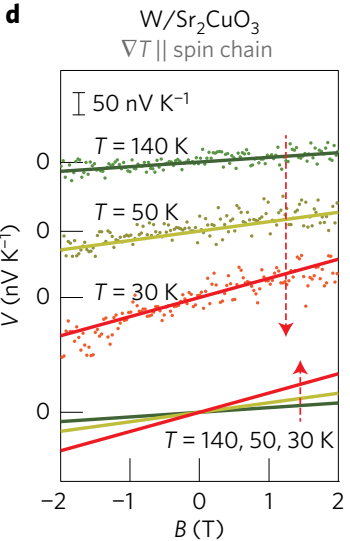

e

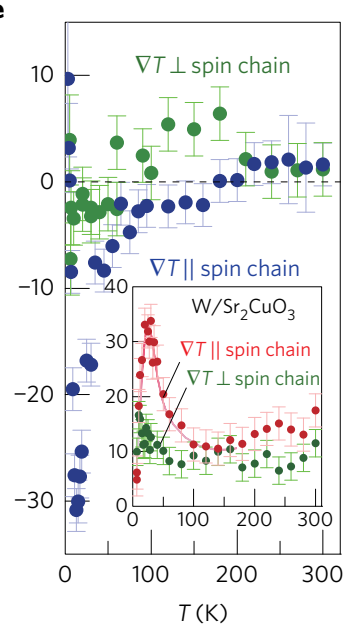

g

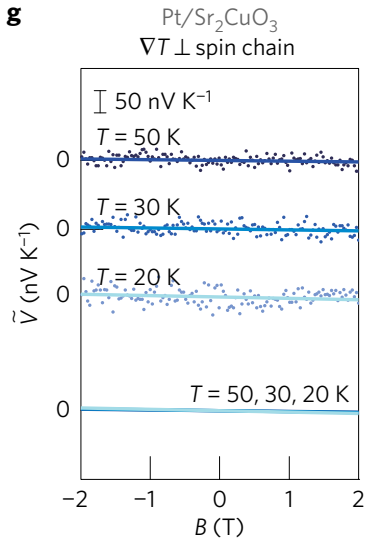

f

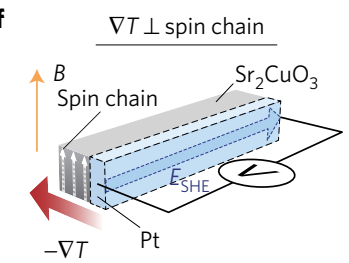

$-\nabla T$
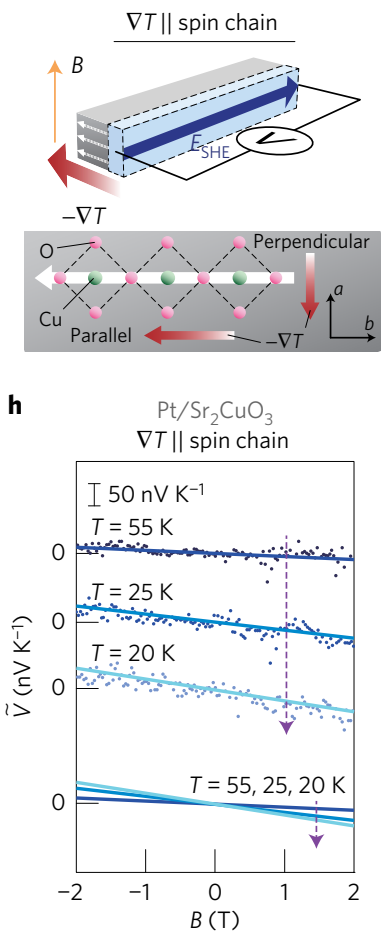

Figure 3 | Spin transport along quantum spin chains. a, $T$ dependence of $\widetilde{V} / B$ measured in $\mathrm{W} / \mathrm{Sr}_{2} \mathrm{CuO}_{3}$. The data for $\mathrm{Pt} / \mathrm{Sr}_{2} \mathrm{CuO} \mathrm{O}_{3}$ are also presented for comparison. The error bars represent the $68 \%$ confidence level ( \pm s.d.). b. Experimental set-ups for $\mathrm{W} / \mathrm{Sr}_{2} \mathrm{CuO}_{3}$ (upper panel) and Pt/Sr $2 \mathrm{CuO} 3($ lower panel). The directions of $E_{\mathrm{SHE}}$ are opposite between $\mathrm{W}$ and $\mathrm{Pt}$ as highlighted by the red and blue arrows. $\mathbf{c}, \mathbf{d}, \mathrm{B}$ dependence of $\widetilde{V}$ measured in $\mathrm{W} / \mathrm{Sr}_{2} \mathrm{CuO} \mathrm{O}_{3}$ at various temperatures. The lines are fits to the data points. e, $T$ dependence of $\widetilde{V} / B$ under $\nabla T$ perpendicular to the spin chains ( $a$ axis, green). The result for $\nabla T$ along the spin chains ( $b$ axis, blue) is shown for comparison. The inset shows the data for $\mathrm{W} / \mathrm{Sr}_{2} \mathrm{CuO}_{3}$. The error bars represent the $68 \%$ confidence level ( \pm s.d.). f, Experimental set-ups for measuring the chain-direction dependence of voltage. The bottom panel shows the directions of $\nabla T$ with respect to the $\mathrm{Cu}-\mathrm{O}$ chains. $\mathbf{g}, \mathbf{h}, B$ dependence of $\widetilde{V}$ measured under $\nabla T$ along the $a$ axis $(\mathbf{g})$ and along the $b$ axis $(\mathbf{h})$ in $\mathrm{Pt}_{/} / \mathrm{Sr}_{2} \mathrm{CuO}_{3}$. The lines are fits to the data points.

susceptibility $X^{-+}(\omega)$. In low-dimensional transport phenomena such as the present spin-chain LSSE, an effect of disorder ${ }^{29}$ is generally relevant in the low-energy limit, while LSSE is a phenomenon driven by a finite temperature and large thermal conduction carried by spinons was observed in $\mathrm{Sr}_{2} \mathrm{CuO}_{3}$ (refs 17,18). These allow us to omit disorder effects within the framework of practical approximation.

Since the metal susceptibility $\chi^{-+}$is well described by a diffusion type function proportional to $\omega$ (ref. 28), computation of $X^{-+}$is the remaining task to obtain the accurate spin current. The low-energy physics with strong singlet correlation of a spin-1/2 AF chain is captured by the TL liquid theory ${ }^{4}$. In the low-energy region, it enables us to calculate $X^{-+}$without any fitting parameters. However, the computed susceptibility is an odd function of $\omega$ even at finite $B$ because of the linearized spinon dispersion. As a result, the linearized standard TL liquid theory leads to zero spin current. To obtain the accurate value of spin current, we consider the actual spinon dispersion beyond the linear approximation. In fact, the spinon dispersion is known to be curved at finite frequencies ${ }^{30}$ and LSSE is driven by finite-frequency spinons. Combining the curved dispersion with the TL liquid theory, we obtain a finite spin current, shown in Fig. 4. In Fig. 4, we also show a result for a ferromagnetic LSSE obtained by calculating the spin current $I_{\mathrm{s}}$ for a three-dimensional ferromagnet (see Supplementary Section C). The spinon $I_{\mathrm{s}}$ is found to be $B$-linear

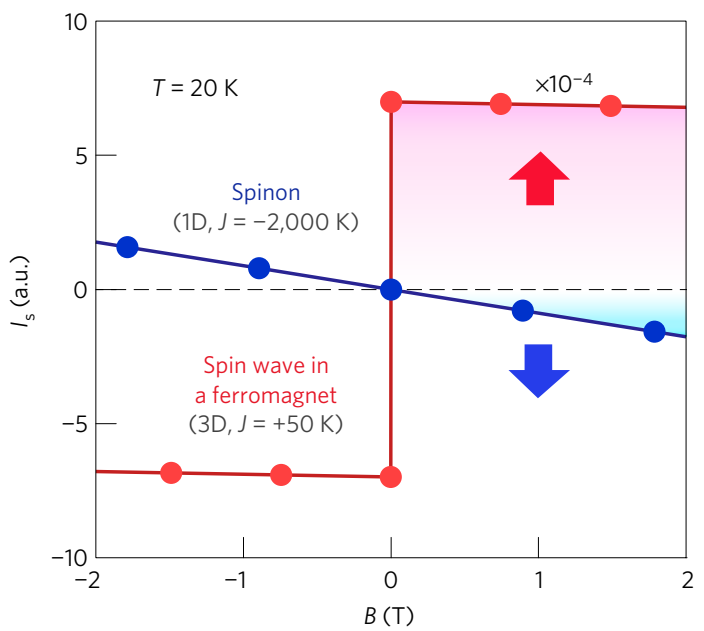

Figure 4 | Theoretical calculations for spinon and ferromagnetic spin Seebeck effects (SSE). $B$ dependence of spin current $\left(I_{s}\right)$ generated via spinon SSE and ferromagnetic SSE. For the spinon (ferromagnetic) SSE calculation, exchange coupling, $J$, was set at $-2,000 \mathrm{~K}(+50 \mathrm{~K})$, which is a typical value of $\mathrm{Sr}_{2} \mathrm{CuO}_{3}\left(\mathrm{Y}_{3} \mathrm{Fe}_{5} \mathrm{O}_{12}\right)$. A sample temperature, $T$, was set at $20 \mathrm{~K}$. The amplitude of the ferromagnetic $I_{\mathrm{s}}$ is multiplied by $10^{-4}$. 
and the linearity continues up to very high fields $(\sim 100 \mathrm{~T})$ owing to the robust gapless excitation of spinons with the large exchange coupling $(\sim 2,000 \mathrm{~K})$; consistent with our experimental results (see also the inset to Fig. 2 d, confirming the $B$-linearity up to 9 T). Most importantly, the sign of the spinon $I_{s}$ is opposite to that of the spinwave $I_{s}$, which captures the marked feature observed experimentally. This sign difference is attributed to whether the dominant weight of $\operatorname{Im} X^{-+}(\omega)$ is located in the positive or negative $\omega$ region (see Supplementary Sections B-D). Since the weight of $\operatorname{Im} X^{-+}(\omega)$ in the positive (negative) $\omega$ region corresponds to down-spin (up-spin) carriers, the sign difference is due to the opposite spin polarization carried by net spin carriers.

\section{Methods}

Methods, including statements of data availability and any associated accession codes and references, are available in the online version of this paper.

\section{Received 3 March 2016; accepted 26 August 2016;} published online 26 September 2016

\section{References}

1. Diep, H. T. (ed.) Frustrated Spin Systems (World Scientific, 2013).

2. Anderson, P. W. The resonating valence bond state in $\mathrm{La}_{2} \mathrm{CuO}_{4}$ and superconductivity. Science 235, 1196-1198 (1987).

3. Wen, X. G. Quantum Field Theory of Many-Body Systems: From the Origin of Sound to an Origin of Light and Electrons (Oxford Univ. Press, 2004).

4. Giamarchi, T. Quantum Physics in One Dimension (Clarendon, 2004)

5. Uchida, K. et al. Observation of the spin Seebeck effect. Nature 455, 778-781 (2008).

6. Jaworski, C. M. et al. Observation of the spin-Seebeck effect in a ferromagnetic semiconductor. Nat. Mater. 9, 898-903 (2010).

7. Uchida, K. et al. Observation of longitudinal spin-Seebeck effect in magnetic insulators. Appl. Phys. Lett. 97, 172505 (2010).

8. Kikkawa, T. et al. Separation of longitudinal spin Seebeck effect from anomalous Nernst effect: determination of origin of transverse thermoelectric voltage in metal/insulator junctions. Phys. Rev. Lett. 88, 214403 (2013).

9. Rezende, S. M. et al. Magnon spin-current theory for the longitudinal spin-Seebeck effect. Phys. Rev. B 89, 014416 (2014).

10. Kikkawa, T. et al. Critical suppression of spin Seebeck effect by magnetic fields. Phys. Rev. B 92, 064413 (2015).

11. Slonczewski, J. C. Current-driven excitation of magnetic multilayers. J. Magn Magn. Mater. 159, L1-L7 (1996).

12. Berger, L. Emission of spin waves by a magnetic multilayer traversed by a current. Phys. Rev. B 54, 9353-9358 (1996).

13. Zutić, I., Fabian, J. \& Das Sarma, S. Spintronics: fundamentals and applications. Rev. Mod. Phys. 76, 323410 (2004).

14. Kajiwara, Y. et al. Transmission of electrical signals by spin-wave interconversion in a magnetic insulator. Nature 464, 262-266 (2010).

15. Cornelissen, L. J., Liu, J., Duine, R. A., Ben Youssef, J. \& van Wees, B. J. Long-range transport of magnon spin information in a magnetic insulator at room temperature. Nat. Phys. 11, 1022-1026 (2015).

16. Motoyama, N., Eisaki, H. \& Uchida, S. Magnetic susceptibility of ideal spin $1 / 2$ Heisenberg antiferromagnetic chain systems, $\mathrm{Sr}_{2} \mathrm{CuO}_{3}$ and $\mathrm{SrCuO}_{2}$. Phys. Rev. Lett. 76, 3212-3215 (1996).
17. Sologubenko, A. V., Giannò, K. \& Ott, H. R. Heat transport by lattice and spin excitations in the spin-chain compounds $\mathrm{SrCuO}_{2}$ and $\mathrm{Sr}_{2} \mathrm{CuO}_{3}$. Phys. Rev. B 64, 054412 (2001)

18. Kawamata, T. et al. Evidence for ballistic thermal conduction in the one-dimensional $S=1 / 2$ Heisenberg antiferromagnetic spin system $\mathrm{Sr}_{2} \mathrm{CuO}_{3}$. J. Phys. Soc. Jpn 77, 034607 (2008).

19. Saitoh, E., Ueda, M., Miyajima, H. \& Tatara, G. Conversion of spin current into charge current at room temperature: inverse spin-Hall effect. Appl. Phys. Lett. 88, 182509 (2006).

20. Valenzuela, S. O. \& Tinkham, M. Direct electronic measurement of the spin Hall effect. Nature 442, 176-179 (2006).

21. Kimura, T., Otani, Y., Sato, T., Takahashi, S. \& Maekawa, S. Room-temperature reversible spin Hall effect. Phys. Rev. Lett. 98, 156601 (2007).

22. Wu, S. M., Pearson, J. E. \& Bhattacharya, A. Paramagnetic spin Seebeck effect. Phys. Rev. Lett. 114, 186602 (2015).

23. Seki, S. et al. Thermal generation of spin current in an antiferromagnet. Phys. Rev. Lett. 115, 266601 (2015).

24. Hoffmann, A. Spin Hall effects in metals. IEEE Trans. Magn. 49, 5172-5193 (2013)

25. Hlubek, N. et al. Spinon heat transport and spin-phonon interaction in the spin-1/2 Heisenberg chain cuprates $\mathrm{Sr}_{2} \mathrm{CuO}_{3}$ and $\mathrm{SrCuO}_{2}$. J. Stat. Mech. 3, P03006 (2012).

26. Boona, S. R. \& Heremans, J. P. Magnon thermal mean free path in yttrium iron garnet. Phys. Rev. B 90, 064421 (2014).

27. Geprägs, S. et al. Origin of the spin Seebeck effect in compensated ferrimagnets. Nat. Commun. 7, 10452 (2015)

28. Adachi, H., Ohe, J., Takahashi, S. \& Maekawa, S. Linear-response theory of spin Seebeck effect in ferromagnetic insulators. Phys. Rev. B 83, 094410 (2011).

29. Giamarchi, T. \& Schulz, H. J. Anderson localization and interactions in one-dimensional metals. Phys. Rev. B 37, 325-340 (1988).

30. Müller, G., Thomas, H., Beck, H. \& Bonner, J. C. Quantum spin dynamics of the antiferromagnetic linear chain in zero and nonzero magnetic field. Phys. Rev. B 24, 1429-1467 (1981)

\section{Acknowledgements}

We thank Y. Ohnuma, N. Yokoi and K. Sato for valuable discussions. This work is supported by ERATO-JST 'Spin Quantum Rectification Project', Japan, Grant-in-Aid for Scientific Research on Innovative Area 'Nano Spin Conversion Science' (No. 26103005), PRESTO ‘Phase Interfaces for Highly Efficient Energy Utilization' from JST, Japan, Grant-in-Aid for Challenging Exploratory Research (No. 16K13827), and Grant-in-Aid for Scientific Research (A) (No. 15H02012) from MEXT, Japan. D.H. is supported by the Yoshida Scholarship Foundation through the Doctor 21 programme.

\section{Author contributions}

D.H. and E.S. designed the experiments; T.K. grew single crystals used in the study; D.H. collected and analysed the data; K.-i.U. and R.I. supported the experiments; M.S. developed the theoretical explanations; S.M., Y.K. and E.S. supervised the study; D.H., M.S., Y.S. and E.S. wrote the manuscript. All authors discussed the results and commented on the manuscript.

\section{Additional information}

Supplementary information is available in the online version of the paper. Reprints and permissions information is available online at www.nature.com/reprints. Correspondence and requests for materials should be addressed to D.H. or E.S.

\section{Competing financial interests}

The authors declare no competing financial interests. 


\section{Methods}

Sample preparation. The single-crystalline $\mathrm{Sr}_{2} \mathrm{CuO}_{3}$ was grown from primary compounds $\mathrm{SrCO}_{3}$ and $\mathrm{CuO}$ with $99.999 \%$ by a travelling-solvent floating-zone method ${ }^{18}$. The single-crystalline $\mathrm{Sr}_{2} \mathrm{CuO}_{3}$ was cut into a cuboid $5 \mathrm{~mm}$ long, $1 \mathrm{~mm}$ wide and $1 \mathrm{~mm}$ thick. The surface of the $\mathrm{Sr}_{2} \mathrm{CuO}_{3}$ was polished mechanically in a glove box filled with $\mathrm{N}_{2}$ gas. We found that exposure of the sample to air causes deterioration of the sample, since the surface of $\mathrm{Sr}_{2} \mathrm{CuO}_{3}$ reacts rapidly with moisture ${ }^{25}$. The 7-nm-thick Pt film was then sputtered on the polished surface $\left(5 \times 1 \mathrm{~mm}^{2}\right)$ of the $\mathrm{Sr}_{2} \mathrm{CuO}_{3}$ in an Ar atmosphere.

Voltage measurement. Voltage data were taken in a Physical Properties Measurement System (Quantum Design). The $\mathrm{Pt}(\mathrm{W}) / \mathrm{Sr}_{2} \mathrm{CuO}_{3}$ sample was sandwiched by sapphire plates and the bottom of the sample was thermally anchored at the system temperature $T$. The temperature gradient $\nabla T$ was generated by applying a charge current to a chip resistor $(100 \Omega)$ on the sapphire plate attached to the metal film. The temperature difference $\Delta T$ between the sapphire plates was set to be $\Delta T / T<0.1$ at each system temperature $T$. Two electrodes were attached to both the ends of the metal film to measure the voltage. An external magnetic field was applied normal to the direction of $\nabla T$ as well as the direction across the two electrodes.

Data availability. The data that support the plots within this paper and other findings of this study are available from the corresponding authors on request. 